Tropical Journal of Pharmaceutical Research February 2016; 15 (2): 393-404

ISSN: $1596-5996$ (print); 1596-9827 (electronic) (c) Pharmacotherapy Group, Faculty of Pharmacy, University of Benin, Benin City, 300001 Nigeria.

All rights reserved.

\title{
Synthesis and Evaluation of Antimicrobial Activity of Some 2-Morpholinomethylamino-4-(7-Unsubstituted/Substituted Coumarin-3-yl)-6-Chlorosubstitutedphenyl Pyrimidines
}

\author{
Mohd Imran $^{1^{*}}$, Abida ${ }^{1}$ and Abdulkhaliq J Alsalman ${ }^{2}$ \\ ${ }^{1}$ Department of Pharmaceutical Chemistry, ${ }^{2}$ Department of Clinical Pharmacy, Faculty of Pharmacy, Northern Border \\ University, Rafha, 91911, PO Box 840, Kingdom of Saudi Arabia
}

*For correspondence: Email: imran_inderlok@yahoo.co.in; Tel: +966535129629

Received: 26 September 2015

Revised accepted: 9 January 2016

\begin{abstract}
Purpose: To prepare some 2-morpholinomethylamino-4-(7-unsubstituted/substitutedcoumarin-3-yl)-6chlorosubstitutedphenyl pyrimidines as antimicrobial agents.

Methods: Some 2-morpholinomethylamino-4-(7-unsubstituted/substitutedcoumarin-3-yl)-6-chlorosubstitutedphenyl pyrimidines were prepared by reacting 2-amino-4-(7-substituted/unsubstituted coumarin-3-yl)-6-(chlorosubstitutedphenyl) pyrimidines with morpholine and formaldehyde. The chemical structures of the synthesized compounds were elucidated by their Fourier Transform infra-red (FTIR), ${ }^{1} \mathrm{H}$-nuclear magnetic resonance $\left({ }^{1} \mathrm{H}\right.$-NMR) and mass spectra, as well as by elemental analysis. These compounds were investigated for their antimicrobial activity against ten bacteria and five fungi by serial plate dilution method using the standard drugs, ofloxacin and ketoconazole, respectively, and their minimum inhibitory concentrations (MICs) were determined.

Results: A total of eighteen new compounds (1a-18a) were synthesized. Compound 3a (MIC $=75$ $\mu \mathrm{g} / \mathrm{mL} ; p<0.0001)$ and $15 \mathrm{a}(\mathrm{MIC}=125 \mu \mathrm{g} / \mathrm{mL} ; p<0.001)$ produced stronger antifungal activity than the standard drug, ketoconazole (MIC $=25 \mu \mathrm{g} / \mathrm{mL} ; p<0.0001)$ against P. citrinum. Compound $4 a$ displayed higher but moderate activity against Gram-positive bacterium, S. aureus (MIC $=100 \mu \mathrm{g} / \mathrm{mL} ; p$ $<0.05$ ) than the standard drug, ofloxacin (MIC $=25 \mu \mathrm{g} / \mathrm{mL} ; p<0.0001)$. Compound 4a also displayed higher but moderate activity against the Gram-negative bacterium, E. coli (MIC $=75 \mu \mathrm{g} / \mathrm{mL} ; p<0.0001)$ than the standard drug, ofloxacin $(M I C=12.5 \mu \mathrm{g} / \mathrm{mL} ; p<0.0001)$. The structure activity relationship analysis revealed that the chloro- substitution at position 2 of the phenyl ring along with a chlorobromosubstituted coumarin moiety of the synthesized compounds is critical for activity against Gram-positive bacteria, Gram negative bacteria and fungi.

Conclusion: The synthesized compounds are relatively active antifungal agents but are weak antibacterial agents. However, they require further evaluation of their antifungal activity against other fungal strains to ascertain their broad spectrum activity.
\end{abstract}

Keywords: Pyrimidine, Coumarin, Morpholine, Antibacterial, Antifungal, Structure-activity relationship

Tropical Journal of Pharmaceutical Research is indexed by Science Citation Index (SciSearch), Scopus, International Pharmaceutical Abstract, Chemical Abstracts, Embase, Index Copernicus, EBSCO, African Index Medicus, JournalSeek, Journal Citation Reports/Science Edition, Directory of Open Access Journals (DOAJ), African Journal Online, Bioline International, Open-J-Gate and Pharmacy Abstracts

\section{INTRODUCTION}

Microbial infections have been creating problems for mankind since centuries and scientists have also developed a large number of antimicrobial agents for the treatment of these infections. According to one new report, about 40 new microbial diseases have been identified since 1970s and more than 2 million Americans are suffering from antibiotic resistance, of which 
about 23,000 die each year [1]. Because of the development of antibiotic resistance and emergence of new microbial diseases, there is a need to develop new antimicrobial agents for the treatment of microbial infections.

Pyrimidine derivatives have an important place in medicinal chemistry as these are associated with a broad range of biological activities [2-4] including antioxidant activity [5-7] and antimicrobial activity [8-13]. The clinical importance of pyrimidine nucleus is also evident by the marketing of clinically used pyrimidine derivatives as well as fused pyrimidine derivatives, for example, as antineoplastic agent (Tegafur), as vasodilator (Dipyridamole), as expectorant (Tasuldine) and as antibacterial agent (Trimethoprim, Piromidic Acid, Tetroxoprim, Metioprim), as antifungal agent (Flucytosine), and as antiviral agent (Broxuridine, Idoxuridine) [14]. Recently, the significance and biological importance of pyrimidine derivatives including their clinical applications in the microbial world has been reviewed [15]. The antimicrobial activity of pyrimidine derivatives against broad range of microbes makes it an important skeleton in medicinal chemistry and drug development against microbes. Morpholine nucleus is an integral part of linezolid, a clinically used drug for the treatment of infections caused by gram-positive bacteria [16]. A number of morpholine containing chemical compounds have also been reported as antimicrobial agents [17-22]. Encouraged by these observations and also in continuation of our search for potent antimicrobial agents [23,24] including antimicrobial agents having coumarin moiety $[25,26]$, we decided to prepare some 2morpholinomethylamino-4-(7-

unsubstituted/substitutedcoumarin-3-yl)-6-

chlorosubstitutedphenyl pyrimidines, herein after the title compounds (1a-18a), as antimicrobial agents.

\section{EXPERIMENTAL}

\section{General}

Melting points were measured in open capillary tubes and are uncorrected. IR $(\mathrm{KBr})$ spectra were recorded on a Nicolet, 5PC FT-IR spectrometer (Browser Morner, USA) and ${ }^{1} \mathrm{H}-\mathrm{NMR}$ spectra on a Bruker DRX-300 FT NMR (Bruker, Germany) spectrophotometer using TMS as internal reference (chemical shift in $\delta \mathrm{ppm}$ ). Mass spectra were recorded on a Jeol-JMS-D-300 mass spectrometer (70 eV) (Jeol, Japan). Satisfactory analysis for $\mathrm{C}, \mathrm{H}$, and $\mathrm{N}$ was obtained for the compounds within $\pm 0.4 \%$ of the theoretical values. Purity of the compounds was checked on silica gel $G$ plates using iodine vapours as visualizing agent. $R_{f}$ value of the compounds was determined by using a mixture of benzene and acetone $(9: 1)$. All reagents used in the present work were of analytical grade. The synthetic pathway for the preparation of the title compounds (1a-18a) is provided in Fig. 1.

The 2-amino-4-(7-substituted/unsubstituted coumarin-3-yl)-6-(chlorosubstitutedphenyl)

pyrimidines (1-18) prepared according to our previous report [25] were reacted with morpholine and formaldehyde in absolute ethanol to provide the title compounds (1a-18a).

\section{General method for the synthesis (1a-18a)}

A mixture of 2-amino-4-(7-unsubstituted/ substituted coumarin-3-yl)-6-chlorosubstituted phenyl pyrimidines ( 0.01 mole), morpholine $(0.01$ mole) and formaldehyde (0.015 moles) was refluxed in absolute ethanol for 6 to $10 \mathrm{~h}$. The reaction mixture was reduced to half of its volume and poured on crushed ice. The solid separated was filtered, washed with water repeatedly, dried and recrystallized from ethanol.

\section{Evaluation of antimicrobial activity}

The title compounds (1a-18a) were tested for their in vitro antimicrobial activity by serial plate dilution method [27,28] against Gram-positive bacteria, Staphylococcus aureus (ATCC 25923), Enterococcus faecalis (ATCC 29212), Staphylococcus epidermidis (ATCC 12228), Bacillus subtilis (ATCC 6633) and Bacillus cereus (ATCC 9946); Gram-negative bacteria, Escherichia coli (ATCC 25922), Pseudomonas aeruginosa (ATCC 27853), Klebsiella pneumoniae (ATCC 700603), Bordetella bronchiseptica (ATCC 4617) and Proteus vulgaris (ATCC 9920); fungi, Candida albicans (ATCC 2091), Aspergillus niger (MTCC 281), Aspergillus flavus (MTCC 277), Monascus purpureous (MTCC 369) and Penicillium citrinum (NCIM 768).

The microorganisms were obtained from the Department of Microbiology, Majeedia Hospital, New Delhi, India. The Department of Microbiology of Majeedia Hospital obtained some of these microorganisms from the Institute of Genomics and Integrative Biology, New Delhi, India. 
<smiles>[X]c1ccc(C=O)c(O)c1</smiles>

(A)<smiles>CCOC(=O)CC(C)=O</smiles>

(B)<smiles>[X]c1ccc2cc(-c3cc([Al])nc(N)n3)c(=O)oc2c1</smiles>

$(1-18)$

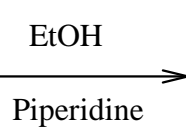<smiles>[X]c1ccc2cc(C(C)=O)c(=O)oc2c1</smiles>

Guanidine Carbonate

$\mathrm{EtOH}$<smiles>[X]c1ccc2cc(C(=O)/C=C/[AlH2+])c(=O)oc2c1</smiles>

$\mathrm{X}=\mathrm{H}, \mathrm{Cl}, \mathrm{Br}$

$\mathrm{Ar}=$ 2-chlorophenyl

4-chlorophenyl

2,6-dichlorophenyl

2,4-dichlorophenyl

2,5-dichlorophenyl

3,5-dichlorophenyl

3,4-dichlorophenyl

Fig. 1. Synthesis of the title compounds (1a-18a)

Nutrient agar medium and Sabouraud dextrose medium were used for antibacterial activity and antifungal activity, respectively. The compounds were tested at concentrations of $200,175,150$, $125,100,75,50,25$ and $12.5 \mu \mathrm{g} / \mathrm{mL}$. The reference or standard antibiotics, ofloxacin and ketoconazole, were used at 50, 25 and 12.5 $\mu \mathrm{g} / \mathrm{mL}$ concentrations for antibacterial activity and antifungal activity, respectively. Sterile dimethyl sulfoxide (DMSO) was used for the preparation of desired concentrations of the synthesized compounds and standard antibiotics. Sterile dimethyl sulfoxide without the synthesized compounds and standard antibiotics served as control group. The minimum inhibitory concentrations (MICs) values of the synthesized compounds, ofloxacin and ketoconazole, were also determined. The minimum inhibitory concentration (MIC) has been defined as the lowest concentration of a compound that inhibited visible growth of microorganisms on the plate.

\section{Statistical analysis}

All antimicrobial activity data are presented as mean $\pm \operatorname{SEM}(n=6)$. The data were analyzed by one-way analysis of variance (ANOVA) with Dunnett's Multiple Comparison Test with respect to control group and standard groups using GraphPad Prism version 5.00 for Windows (GraphPad Software, San Diego California USA). The results were considered significantly different at $p<0.05$.

\section{RESULTS}

The title compounds (1a-18a) were prepared according to the method outlined in Fig 1. The characterization data of the intermediates, (C), (D) and (1-18) of the Fig. 1 were in line with our previously published data $[25,26]$. The structures of the title compounds (1a-18a) were confirmed on the basis of their IR, ${ }^{1} \mathrm{H}-\mathrm{NMR}$, Mass and elemental analysis data. The appearance of the 
IR absorption peaks from $3290 \mathrm{~cm}^{-1}$ to $3280 \mathrm{~cm}^{-1}$ confirmed the stretching vibration of $\mathrm{N}-\mathrm{H}$ group of $-\mathrm{NH}-\mathrm{CH}_{2}$ - moiety; from $1710 \mathrm{~cm}^{-1}$ to $1705 \mathrm{~cm}^{-1}$ confirmed the stretching vibration of $\mathrm{C}=\mathrm{O}$ group of the coumarin moiety; from $1611 \mathrm{~cm}^{-1}$ to 1604 $\mathrm{cm}^{-1}$ confirmed the stretching vibration of $\mathrm{C}=\mathrm{N}$ group of the pyrimidine ring; from $1545 \mathrm{~cm}^{-1}$ to $1540 \mathrm{~cm}^{-1}$ confirmed the stretching vibration of $\mathrm{C}=\mathrm{C}$ group of aromatic $\mathrm{C}=\mathrm{C}$ bond; and from $1135 \mathrm{~cm}^{-1}$ to $1130 \mathrm{~cm}^{-1}$ confirmed the stretching vibration of $\mathrm{C}-\mathrm{O}-\mathrm{C}$ group of coumarin moiety and morpholine moieties present in the title compounds (1a-18a). The appearance of the signals in the ${ }^{1} \mathrm{H}$-NMR spectra of the title compounds (1a-18a) at $\delta(\mathrm{ppm})$ values from 2.63 to 2.72 confirmed four protons of $-\mathrm{CH}_{2}-\mathrm{N}-\mathrm{CH}_{2}-$ portion of the morpholine moiety; from 3.50 to 3.58 confirmed four protons of $-\mathrm{CH}_{2}-\mathrm{O}-\mathrm{CH}_{2}$ portion of the morpholin moiety; from 4.27 to 4.42 confirmed two methylene protons of $-\mathrm{NH}-\mathrm{CH}_{2}-$ moiety; from 6.90 to 7.75 confirmed the number of aromatic protons; and from 7.80 to 789 confirmed the secondary amino group (exchangeable with $\mathrm{D}_{2} \mathrm{O}$ ) of $-\mathrm{NH}-\mathrm{CH}_{2}$ - moiety of the title compounds (1a-18a). The elemental analysis and molecular ion peaks of the title compounds (1a-18a) were also consistent with the assigned structures. The detailed physical constants, FTIR, ${ }^{1} \mathrm{H}-\mathrm{NMR}$, mass and elemental analysis data of the title compounds (1a-18a) are presented as follows.

\section{2-(Morpholinomethylamino)-4-(coumarin-3- yl)-6-(4-chlorophenyl) pyrimidine (1a)}

Yield: 55 \%; m.p.: 147-149 ${ }^{\circ} \mathrm{C}$; $\mathrm{R}_{\mathrm{f}}$ : 0.76; IR (KBr) $\mathrm{cm}^{-1}: 3289(\mathrm{~N}-\mathrm{H}), 1706(\mathrm{C}=\mathrm{O}), 1607(\mathrm{C}=\mathrm{N}), 1544$ $(\mathrm{C}=\mathrm{C}), 1131$ (C-O-C); 'H-NMR (CDCl 3 , DMSO$\left.\mathrm{d}_{6}\right) \delta$ ppm: $2.66\left(\mathrm{t}, \mathrm{J}=8 \mathrm{~Hz}, 4 \mathrm{H},-\mathrm{CH}_{2}-\mathrm{N}^{-} \mathrm{CH}_{2}-\right)$, $3.52\left(\mathrm{t}, \mathrm{J}=8 \mathrm{~Hz}, 4 \mathrm{H},-\mathrm{CH}_{2}-\mathrm{O}-\mathrm{CH}_{2}-\right), 4.27(\mathrm{~d}, \mathrm{~J}=$

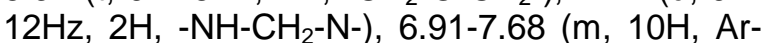
$\mathrm{H}), 7.86\left(\mathrm{~s}, 1 \mathrm{H}, \mathrm{NH}\right.$, exchangable with $\left.\mathrm{D}_{2} \mathrm{O}\right)$; Elemental Analysis $\left(\mathrm{C}_{24} \mathrm{H}_{21} \mathrm{~N}_{4} \mathrm{O}_{3} \mathrm{Cl}\right)$, Found\% (Calculated\%): C, 64.20 (64.21); H, 4.72 (4.72); $\mathrm{N}, 12.47$ (12.48); Mass (m/z): $448\left(\mathrm{M}^{+}\right.$, $\left.\mathrm{C}_{24} \mathrm{H}_{21} \mathrm{~N}_{4} \mathrm{O}_{3} \mathrm{Cl}\right), \quad 449 \quad\left(\mathrm{M}^{+}+1\right), \quad 164 \quad(100 \%$, $\mathrm{C}_{9} \mathrm{H}_{7} \mathrm{NCl}$ ).

\section{2-(Morpholinomethylamino)-4-(coumarin-3- yl)-6-(2,6-dichlorophenyl)pyrimidine (2a)}

Yield: 55\%; m.p.: $140-142{ }^{\circ} \mathrm{C}$; $\mathrm{R}_{\mathrm{f}}$ : 0.74; IR (KBr) $\mathrm{cm}^{-1}: 3287(\mathrm{~N}-\mathrm{H}), 1707(\mathrm{C}=\mathrm{O}), 1611(\mathrm{C}=\mathrm{N}), 1543$ $(\mathrm{C}=\mathrm{C}), 1133$ (C-O-C); 'H-NMR ( $\mathrm{CDCl}_{3}$, DMSO$\left.\mathrm{d}_{6}\right) \delta$ ppm: $2.69\left(\mathrm{t}, \mathrm{J}=8 \mathrm{~Hz}, 4 \mathrm{H},-\mathrm{CH}_{2}-\mathrm{N}^{-} \mathrm{CH}_{2}-\right.$ ), $3.57\left(\mathrm{t}, \mathrm{J}=8 \mathrm{~Hz}, 4 \mathrm{H},-\mathrm{CH}_{2}-\mathrm{O}-\mathrm{CH}_{2}-\right), 4.32(\mathrm{~d}, \mathrm{~J}=$ $\left.12 \mathrm{~Hz}, 2 \mathrm{H},-\mathrm{NH}-\mathrm{CH}_{2}-\mathrm{N}-\right)$, 6.93-7.73 (m, 9H, Ar-H), 7.88 (s, $1 \mathrm{H}, \mathrm{NH}$, exchangable with $\left.\mathrm{D}_{2} \mathrm{O}\right)$; Elemental Analysis $\left(\mathrm{C}_{24} \mathrm{H}_{20} \mathrm{~N}_{4} \mathrm{O}_{3} \mathrm{Cl}_{2}\right)$, Found\%
(Calculated\%): C, 59.62 (59.64); H, 4.17 (4.17); N, 11.58 (11.59).

\section{2-(Morpholinomethylamino)-4-(coumarin-3- yl)-6-(2,4-dichlorophenyl)pyrimidine (3a)}

Yield: 50 \%; m.p.: $155-157^{\circ} \mathrm{C}$; $\mathrm{R}_{\mathrm{f}}$ : 0.71; IR (KBr) $\mathrm{cm}^{-1}$ : $3287(\mathrm{~N}-\mathrm{H}), 1706(\mathrm{C}=\mathrm{O}), 1607(\mathrm{C}=\mathrm{N}), 1542$ $(\mathrm{C}=\mathrm{C}), 1130$ (C-O-C); 'H-NMR $\left(\mathrm{CDCl}_{3}\right.$, DMSO$\left.\mathrm{d}_{6}\right) \delta \mathrm{ppm}: 2.66\left(\mathrm{t}, \mathrm{J}=8 \mathrm{~Hz}, 4 \mathrm{H},-\mathrm{CH}_{2}-\mathrm{N}-\mathrm{CH}_{2}-\right)$, $3.50\left(\mathrm{t}, \mathrm{J}=8 \mathrm{~Hz}, 4 \mathrm{H},-\mathrm{CH}_{2}-\mathrm{O}-\mathrm{CH}_{2}-\right), 4.35(\mathrm{~d}, \mathrm{~J}=$ $\left.12 \mathrm{~Hz}, 2 \mathrm{H},-\mathrm{NH}-\mathrm{CH}_{2}-\mathrm{N}-\right)$, 7.02-7.75 (m, 9H, Ar- $\left.\mathrm{H}\right)$, $7.84\left(\mathrm{~s}, 1 \mathrm{H}, \mathrm{NH}\right.$, exchangable with $\left.\mathrm{D}_{2} \mathrm{O}\right)$; Elemental Analysis $\left(\mathrm{C}_{24} \mathrm{H}_{20} \mathrm{~N}_{4} \mathrm{O}_{3} \mathrm{Cl}_{2}\right)$, Found\% (Calculated\%): C, 59.63 (59.64); H, 4.17 (4.17); $\mathrm{N}, 11.57$ (11.59); Mass (m/z): $483\left(\mathrm{M}^{+}\right.$, $\left.\mathrm{C}_{24} \mathrm{H}_{20} \mathrm{~N}_{4} \mathrm{O}_{3} \mathrm{Cl}_{2}\right), \quad 484 \quad\left(\mathrm{M}^{+}+1\right), \quad 199 \quad(100 \%$, $\mathrm{C}_{9} \mathrm{H}_{6} \mathrm{NCl}_{2}$ ).

\section{2-(Morpholinomethylamino)-4-(coumarin-3- yl)-6-(2-chlorophenyl)pyrimidine (4a)}

Yield: 55 \%; m.p.: $147-149{ }^{\circ} \mathrm{C}$; $\mathrm{R}_{\mathrm{f}}$ : 0.77; IR (KBr) $\mathrm{cm}^{-1}$ : $3286(\mathrm{~N}-\mathrm{H}), 1706(\mathrm{C}=\mathrm{O}), 1605(\mathrm{C}=\mathrm{N}), 1545$ $(\mathrm{C}=\mathrm{C}), 1133$ (C-O-C); 'H-NMR $\left(\mathrm{CDCl}_{3}\right.$, DMSO$\left.\mathrm{d}_{6}\right) \delta$ ppm: $2.68\left(\mathrm{t}, \mathrm{J}=8 \mathrm{~Hz}, 4 \mathrm{H},-\mathrm{CH}_{2}-\mathrm{N}^{-} \mathrm{CH}_{2}-\right)$, 3.55 (t, J = 8Hz, 4H, $\left.-\mathrm{CH}_{2}-\mathrm{O}-\mathrm{CH}_{2}-\right), 4.35$ (d, J =

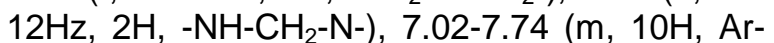
$\mathrm{H}), 7.88\left(\mathrm{~s}, 1 \mathrm{H}, \mathrm{NH}\right.$, exchangable with $\left.\mathrm{D}_{2} \mathrm{O}\right)$; Elemental Analysis $\left(\mathrm{C}_{24} \mathrm{H}_{21} \mathrm{~N}_{4} \mathrm{O}_{3} \mathrm{Cl}\right)$, Found\% (Calculated\%): C, 64.20 (64.21); H, 4.71 (4.72); N, 12.47 (12.48).

\section{2-(Morpholinomethylamino)-4-(7-chlorocou- marin-3-yl)-6-(4-chlorophenyl)pyrimidine (5a)}

Yield: 55 \%; m.p.: $172-174{ }^{\circ} \mathrm{C}$; $\mathrm{R}_{\mathrm{f}}$ : 0.69; IR ( $\left.\mathrm{KBr}\right)$ $\mathrm{cm}^{-1}$ : $3280(\mathrm{~N}-\mathrm{H}), 1705(\mathrm{C}=\mathrm{O}), 1607(\mathrm{C}=\mathrm{N}), 1544$ $(\mathrm{C}=\mathrm{C}), 1130$ (C-O-C); 'H-NMR $\left(\mathrm{CDCl}_{3}\right.$, DMSO$\left.\mathrm{d}_{6}\right) \delta \mathrm{ppm}: 2.69\left(\mathrm{t}, \mathrm{J}=8 \mathrm{~Hz}, 4 \mathrm{H},-\mathrm{CH}_{2}-\mathrm{N}-\mathrm{CH}_{2}-\right)$, $3.53\left(\mathrm{t}, \mathrm{J}=8 \mathrm{~Hz}, 4 \mathrm{H},-\mathrm{CH}_{2}-\mathrm{O}-\mathrm{CH}_{2}-\right), 4.35(\mathrm{~d}, \mathrm{~J}=$ $\left.12 \mathrm{~Hz}, 2 \mathrm{H},-\mathrm{NH}-\mathrm{CH}_{2}-\mathrm{N}-\right), 6.90-7.71(\mathrm{~m}, 9 \mathrm{H}, \mathrm{Ar}-\mathrm{H})$, $7.85\left(\mathrm{~s}, 1 \mathrm{H}, \mathrm{NH}\right.$, exchangable with $\left.\mathrm{D}_{2} \mathrm{O}\right)$; Elemental Analysis $\left(\mathrm{C}_{24} \mathrm{H}_{20} \mathrm{~N}_{4} \mathrm{O}_{3} \mathrm{Cl}_{2}\right)$, Found\% (Calculated\%): C, 59.62 (59.64); H, 4.16 (4.17); $\mathrm{N}, 11.57$ (11.59); Mass (m/z): $483\left(\mathrm{M}^{+}\right.$, $\left.\mathrm{C}_{24} \mathrm{H}_{20} \mathrm{~N}_{4} \mathrm{O}_{3} \mathrm{Cl}_{2}\right), \quad 484 \quad\left(\mathrm{M}^{+}+1\right), \quad 164 \quad(100 \%$, $\mathrm{C}_{9} \mathrm{H}_{7} \mathrm{NCl}$ ).

\section{2-(Morpholinomethylamino)-4-(7-bromocou-} marin-3-yl)-6-(4-chlorophenyl)pyrimidine (6a)

Yield: 60 \%; m.p.: $177-179{ }^{\circ} \mathrm{C}$; $\mathrm{R}_{\mathrm{f}}$ : 0.66; IR (KBr) $\mathrm{cm}^{-1}: 3287(\mathrm{~N}-\mathrm{H}), 1710(\mathrm{C}=\mathrm{O}), 1608(\mathrm{C}=\mathrm{N})$, $1542(\mathrm{C}=\mathrm{C}), 1132$ (C-O-C); ${ }^{1} \mathrm{H}-\mathrm{NMR}\left(\mathrm{CDCl}_{3}\right.$, DMSO- $\left.\mathrm{d}_{6}\right) \delta$ ppm: $2.70\left(\mathrm{t}, \mathrm{J}=8 \mathrm{~Hz}, 4 \mathrm{H},-\mathrm{CH}_{2}-\mathrm{N}-\right.$ $\mathrm{CH}_{2}-$ ), 3.54 (t, $\left.\mathrm{J}=8 \mathrm{~Hz}, 4 \mathrm{H},-\mathrm{CH}_{2}-\mathrm{O}-\mathrm{CH}_{2}-\right), 4.39$ $\left(\mathrm{d}, \mathrm{J}=12 \mathrm{~Hz}, 2 \mathrm{H},-\mathrm{NH}-\mathrm{CH}_{2}-\mathrm{N}-\right)$, 6.90-7.69 (m, $9 \mathrm{H}, \mathrm{Ar}-\mathrm{H}), 7.88(\mathrm{~s}, 1 \mathrm{H}, \mathrm{NH}$, exchangable with $\left.\mathrm{D}_{2} \mathrm{O}\right)$; Elemental Analysis $\left(\mathrm{C}_{24} \mathrm{H}_{20} \mathrm{~N}_{4} \mathrm{O}_{3} \mathrm{ClBr}\right)$, 
Found \% (Calculated\%): C, 54.60 (54.62); H, 3.82 (3.82); N, 10.60 (10.62).

\section{2-(Morpholinomethylamino)-4-(7-chlorocou- mrin-3-yl)-6-(2,6-dichlorophenyl)pyrimidine} (7a)

Yield: 50 \%; m.p.: $185-187^{\circ} \mathrm{C}$; $\mathrm{R}_{\mathrm{f}}$ : 0.69; IR (KBr) $\mathrm{cm}^{-1}: 3290(\mathrm{~N}-\mathrm{H}), 1708(\mathrm{C}=\mathrm{O}), 1608(\mathrm{C}=\mathrm{N})$, $1544(\mathrm{C}=\mathrm{C}), 1131$ (C-O-C); ${ }^{1} \mathrm{H}-\mathrm{NMR} \quad\left(\mathrm{CDCl}_{3}\right.$, DMSO- $\left.\mathrm{d}_{6}\right) \delta$ ppm: $2.71\left(\mathrm{t}, \mathrm{J}=8 \mathrm{~Hz}, 4 \mathrm{H},-\mathrm{CH}_{2}-\mathrm{N}-\right.$ $\left.\mathrm{CH}_{2}-\right), 3.53\left(\mathrm{t}, \mathrm{J}=8 \mathrm{~Hz}, 4 \mathrm{H},-\mathrm{CH}_{2}-\mathrm{O}-\mathrm{CH}_{2}-\right), 4.42$ $\left(\mathrm{d}, \mathrm{J}=12 \mathrm{~Hz}, 2 \mathrm{H},-\mathrm{NH}-\mathrm{CH}_{2}-\mathrm{N}-\right), 7.03-7.73(\mathrm{~m}$, $8 \mathrm{H}, \mathrm{Ar}-\mathrm{H}), 7.88(\mathrm{~s}, 1 \mathrm{H}, \mathrm{NH}$, exchangable with $\left.\mathrm{D}_{2} \mathrm{O}\right)$; Elemental Analysis $\left(\mathrm{C}_{24} \mathrm{H}_{19} \mathrm{~N}_{4} \mathrm{O}_{3} \mathrm{Cl}_{3}\right)$, Found \% (Calculated\%): C, 55.66 (55.67); H, 3.70 (3.70); N, 10.81 (10.82).

\section{2-(Morpholinomethylamino)-4-(7-bromocou-} marin-3-yl)-6-(2,6-dichlorophenyl)pyrimidine (8a)

Yield: $55 \%$; m.p.: $171-173{ }^{\circ} \mathrm{C}$; $\mathrm{R}_{\mathrm{f}}$ : 0.66; IR ( $\left.\mathrm{KBr}\right)$ $\mathrm{cm}^{-1}: 3287(\mathrm{~N}-\mathrm{H}), 1705(\mathrm{C}=\mathrm{O}), 1610(\mathrm{C}=\mathrm{N}), 1543$ $(\mathrm{C}=\mathrm{C}), 1132$ (C-O-C); 'H-NMR ( $\mathrm{CDCl}_{3}$, DMSO$\left.\mathrm{d}_{6}\right) \delta$ ppm: $2.72\left(\mathrm{t}, \mathrm{J}=8 \mathrm{~Hz}, 4 \mathrm{H},-\mathrm{CH}_{2}-\mathrm{N}^{-} \mathrm{CH}_{2}-\right.$ ), $3.50\left(\mathrm{t}, \mathrm{J}=8 \mathrm{~Hz}, 4 \mathrm{H},-\mathrm{CH}_{2}-\mathrm{O}-\mathrm{CH}_{2}-\right), 4.35(\mathrm{~d}, \mathrm{~J}=$ $\left.12 \mathrm{~Hz}, 2 \mathrm{H},-\mathrm{NH}-\mathrm{CH}_{2}-\mathrm{N}-\right)$, 6.99-7.69 (m, 8H, Ar-H), $7.86\left(\mathrm{~s}, 1 \mathrm{H}, \mathrm{NH}\right.$, exchangable with $\left.\mathrm{D}_{2} \mathrm{O}\right)$; Elemental Analysis $\left(\mathrm{C}_{24} \mathrm{H}_{19} \mathrm{~N}_{4} \mathrm{O}_{3} \mathrm{Cl}_{2} \mathrm{Br}\right)$, Found \% (Calculated\%): C, 51.25 (51.27); H, 3.40 (3.41); $\mathrm{N}, \quad 9.95$ (9.96); Mass (m/z): $562\left(\mathrm{M}^{+}\right.$, $\left.\mathrm{C}_{24} \mathrm{H}_{19} \mathrm{~N}_{4} \mathrm{O}_{3} \mathrm{Cl}_{2} \mathrm{Br}\right), \quad 564 \quad\left(\mathrm{M}^{+}+2\right), \quad 199$ (100\%, $\mathrm{C}_{9} \mathrm{H}_{6} \mathrm{NCl}_{2}$ ).

\section{2-(Morpholinomethylamino)-4-(7-chlorocou-} marin-3-yl)-6-(2,4-dichlorophenyl)pyrimidine (9a)

Yield: $55 \%$; m.p.: $173-175{ }^{\circ} \mathrm{C}$; $\mathrm{R}_{\mathrm{f}}$ : 0.72; IR ( $\left.\mathrm{KBr}\right)$ $\mathrm{cm}^{-1}: 3287(\mathrm{~N}-\mathrm{H}), 1706(\mathrm{C}=\mathrm{O}), 1604(\mathrm{C}=\mathrm{N})$, $1544(\mathrm{C}=\mathrm{C}), 1130$ (C-O-C); ${ }^{1} \mathrm{H}-\mathrm{NMR}\left(\mathrm{CDCl}_{3}\right.$, DMSO- $\left.\mathrm{d}_{6}\right) \delta \mathrm{ppm}: 2.70\left(\mathrm{t}, \mathrm{J}=8 \mathrm{~Hz}, 4 \mathrm{H},-\mathrm{CH}_{2}-\mathrm{N}-\right.$ $\mathrm{CH}_{2}-$ ), 3.51 (t, $\left.\mathrm{J}=8 \mathrm{~Hz}, 4 \mathrm{H},-\mathrm{CH}_{2}-\mathrm{O}-\mathrm{CH}_{2^{-}}\right), 4.35$ $\left(\mathrm{d}, \mathrm{J}=12 \mathrm{~Hz}, 2 \mathrm{H},-\mathrm{NH}-\mathrm{CH}_{2}-\mathrm{N}-\right)$, 7.03-7.74 (m, $8 \mathrm{H}, \mathrm{Ar}-\mathrm{H}), 7.89(\mathrm{~s}, 1 \mathrm{H}, \mathrm{NH}$, exchangable with $\left.\mathrm{D}_{2} \mathrm{O}\right)$; Elemental Analysis $\left(\mathrm{C}_{24} \mathrm{H}_{19} \mathrm{~N}_{4} \mathrm{O}_{3} \mathrm{Cl}_{3}\right)$, Found \% (Calculated\%): C, 55.66 (55.67); H, 3.70 (3.70); N, 10.82 (10.82).

\section{2-(Morpholinomethylamino)-4-(7-bromocou- marin-3-yl)-6-(2,4-dichlorophenyl)pyrimidine (10a)}

Yield: $60 \%$; m.p.: $176-178{ }^{\circ} \mathrm{C}$; $\mathrm{R}_{\mathrm{f}}$ : 0.71; IR (KBr) $\mathrm{cm}^{-1}: 3280(\mathrm{~N}-\mathrm{H}), 1705(\mathrm{C}=\mathrm{O}), 1605(\mathrm{C}=\mathrm{N})$, $1540(\mathrm{C}=\mathrm{C}), 1133$ (C-O-C); ${ }^{1} \mathrm{H}-\mathrm{NMR}\left(\mathrm{CDCl}_{3}\right.$, DMSO- $\left.\mathrm{d}_{6}\right) \delta$ ppm: $2.71\left(\mathrm{t}, \mathrm{J}=8 \mathrm{~Hz}, 4 \mathrm{H},-\mathrm{CH}_{2}-\mathrm{N}-\right.$ $\left.\mathrm{CH}_{2}-\right), 3.55\left(\mathrm{t}, \mathrm{J}=8 \mathrm{~Hz}, 4 \mathrm{H},-\mathrm{CH}_{2}-\mathrm{O}-\mathrm{CH}_{2}-\right), 4.42$ $\left(\mathrm{d}, \mathrm{J}=12 \mathrm{~Hz}, 2 \mathrm{H},-\mathrm{NH}-\mathrm{CH}_{2}-\mathrm{N}-\right)$, 7.04-7.70 (m, $8 \mathrm{H}, \mathrm{Ar}-\mathrm{H}), 7.88(\mathrm{~s}, 1 \mathrm{H}, \mathrm{NH}$, exchangable with $\left.\mathrm{D}_{2} \mathrm{O}\right)$; Elemental Analysis $\left(\mathrm{C}_{24} \mathrm{H}_{19} \mathrm{~N}_{4} \mathrm{O}_{3} \mathrm{Cl}_{2} \mathrm{Br}\right)$, Found \% (Calculated\%): C, 51.25 (51.27); H, 3.40 (3.41); N, 9.95 (9.96).

\section{2-(Morpholinomethylamino)-4-(7-chlorocou- marin-3-yl)-6-(2-chlorophenyl)pyrimidine (11a)}

Yield: 50 \%; m.p.: $185-187^{\circ} \mathrm{C}$; $\mathrm{R}_{\mathrm{f}}$ : 0.72; IR (KBr) $\mathrm{cm}^{-1}: 3280(\mathrm{~N}-\mathrm{H}), 1705(\mathrm{C}=\mathrm{O}), 1605(\mathrm{C}=\mathrm{N}), 1542$ $(\mathrm{C}=\mathrm{C}), 1130$ (C-O-C); 'H-NMR $\left(\mathrm{CDCl}_{3}\right.$, DMSO$\left.\mathrm{d}_{6}\right) \delta \mathrm{ppm}: 2.69\left(\mathrm{t}, \mathrm{J}=8 \mathrm{~Hz}, 4 \mathrm{H},-\mathrm{CH}_{2}-\mathrm{N}^{-} \mathrm{CH}_{2^{-}}\right)$, 3.58 (t, $\left.\mathrm{J}=8 \mathrm{~Hz}, 4 \mathrm{H},-\mathrm{CH}_{2}-\mathrm{O}-\mathrm{CH}_{2}-\right), 4.37(\mathrm{~d}, \mathrm{~J}=$ $\left.12 \mathrm{~Hz}, 2 \mathrm{H},-\mathrm{NH}-\mathrm{CH}_{2}-\mathrm{N}-\right)$, 7.01-7.74 (m, 9H, Ar-H), $7.86\left(\mathrm{~s}, 1 \mathrm{H}, \mathrm{NH}\right.$, exchangable with $\left.\mathrm{D}_{2} \mathrm{O}\right)$; Elemental Analysis $\left(\mathrm{C}_{24} \mathrm{H}_{20} \mathrm{~N}_{4} \mathrm{O}_{3} \mathrm{Cl}_{2}\right)$, Found \% (Calculated \%): C, 59.63 (59.64); H, 4.16 (4.17); N, 11.58 (11.59).

\section{2-(Morpholinomethylamino)-4-(7-bromocou-} marin-3-yl)-6-(2-chlorophenyl)pyrimidine (12a)

Yield: $55 \%$; m.p.: $176-178{ }^{\circ} \mathrm{C}$; $\mathrm{R}_{\mathrm{f}}$ : 0.76; IR (KBr) $\mathrm{cm}^{-1}: 3280(\mathrm{~N}-\mathrm{H}), 1706(\mathrm{C}=\mathrm{O}), 1606(\mathrm{C}=\mathrm{N})$, $1544(\mathrm{C}=\mathrm{C}), 1130$ (C-O-C); ${ }^{1} \mathrm{H}-\mathrm{NMR}\left(\mathrm{CDCl}_{3}\right.$, DMSO- $\left.\mathrm{d}_{6}\right) \delta$ ppm: $2.70\left(\mathrm{t}, \mathrm{J}=8 \mathrm{~Hz}, 4 \mathrm{H},-\mathrm{CH}_{2}-\mathrm{N}-\right.$ $\left.\mathrm{CH}_{2}-\right), 3.56\left(\mathrm{t}, \mathrm{J}=8 \mathrm{~Hz}, 4 \mathrm{H},-\mathrm{CH}_{2}-\mathrm{O}-\mathrm{CH}_{2}-\right), 4.35$ $\left(\mathrm{d}, \mathrm{J}=12 \mathrm{~Hz}, 2 \mathrm{H},-\mathrm{NH}-\mathrm{CH}_{2}-\mathrm{N}-\right)$, 7.02-7.69 (m, $9 \mathrm{H}, \mathrm{Ar}-\mathrm{H}), 7.88(\mathrm{~s}, 1 \mathrm{H}, \mathrm{NH}$, exchangable with $\left.\mathrm{D}_{2} \mathrm{O}\right)$; Elemental Analysis $\left(\mathrm{C}_{24} \mathrm{H}_{20} \mathrm{~N}_{4} \mathrm{O}_{3} \mathrm{ClBr}\right)$, Found \% (Calculated \%): C, 54.61 (54.62); $\mathrm{H}$, 3.81 (3.82); N, 10.60 (10.62); Mass (m/z): 527 $\left(\mathrm{M}^{+}, \mathrm{C}_{24} \mathrm{H}_{20} \mathrm{~N}_{4} \mathrm{O}_{3} \mathrm{ClBr}\right), 529\left(\mathrm{M}^{+}+2\right), 164(100 \%$, $\mathrm{C}_{9} \mathrm{H}_{7} \mathrm{NCl}$ ).

2-(Morpholinomethylamino)-4-(7-chlorocoumarin-3-yl)-6-(2,5-dichlorophenyl)pyrimidine (13a)

Yield: 45 \%; m.p.: $190-192{ }^{\circ} \mathrm{C}$; $\mathrm{R}_{\mathrm{f}}$ : 0.82; IR (KBr) $\mathrm{cm}^{-1}: 3288(\mathrm{~N}-\mathrm{H}), 1707(\mathrm{C}=\mathrm{O}), 1609(\mathrm{C}=\mathrm{N}), 1541$ $(\mathrm{C}=\mathrm{C}), 1132(\mathrm{C}-\mathrm{O}-\mathrm{C})$; ' $\mathrm{H}-\mathrm{NMR}\left(\mathrm{CDCl}_{3}\right.$, DMSO$\left.\mathrm{d}_{6}\right) \delta \mathrm{ppm}: 2.68\left(\mathrm{t}, \mathrm{J}=8 \mathrm{~Hz}, 4 \mathrm{H},-\mathrm{CH}_{2}-\mathrm{N}^{-} \mathrm{CH}_{2}-\right)$, 3.55 (t, J = 8Hz, 4H, $\left.-\mathrm{CH}_{2}-\mathrm{O}-\mathrm{CH}_{2}-\right), 4.35(\mathrm{~d}, \mathrm{~J}=$ $\left.12 \mathrm{~Hz}, 2 \mathrm{H},-\mathrm{NH}-\mathrm{CH}_{2}-\mathrm{N}-\right), 6.95-7.70(\mathrm{~m}, 8 \mathrm{H}, \mathrm{Ar}-\mathrm{H})$, $7.88\left(\mathrm{~s}, 1 \mathrm{H}, \mathrm{NH}\right.$, exchangable with $\left.\mathrm{D}_{2} \mathrm{O}\right)$; Elemental Analysis $\left(\mathrm{C}_{24} \mathrm{H}_{19} \mathrm{~N}_{4} \mathrm{O}_{3} \mathrm{Cl}_{3}\right)$, Found \% (Calculated\%): C, 55.66 (55.67); H, 3.68 (3.70); N, 10.81 (10.82).

\section{2-(Morpholinomethylamino)-4-(7-bromocou-} marin-3-yl)-6-(2,5-dichlorophenyl)pyrimidine (14a)

Yield: 50 \%; m.p.: $210-212{ }^{\circ} \mathrm{C}$; $\mathrm{R}_{f}: 0.75$; IR (KBr) $\mathrm{cm}^{-1}$ : $3283(\mathrm{~N}-\mathrm{H}), 1709(\mathrm{C}=\mathrm{O}), 1611(\mathrm{C}=\mathrm{N}), 1544$ $(\mathrm{C}=\mathrm{C}), 1134(\mathrm{C}-\mathrm{O}-\mathrm{C})$; $\mathrm{H}-\mathrm{NMR}\left(\mathrm{CDCl}_{3}\right.$, DMSO$\left.\mathrm{d}_{6}\right) \delta$ ppm: $2.65\left(\mathrm{t}, \mathrm{J}=8 \mathrm{~Hz}, 4 \mathrm{H},-\mathrm{CH}_{2}-\mathrm{N}^{-} \mathrm{CH}_{2}-\right.$ ), 
$3.51\left(\mathrm{t}, \mathrm{J}=8 \mathrm{~Hz}, 4 \mathrm{H},-\mathrm{CH}_{2}-\mathrm{O}-\mathrm{CH}_{2}-\right), 4.29$ (d, J = $\left.12 \mathrm{~Hz}, 2 \mathrm{H},-\mathrm{NH}-\mathrm{CH}_{2}-\mathrm{N}-\right)$, 6.99-7.72 (m, 8H, Ar-H), 7.82 (s, $1 \mathrm{H}, \mathrm{NH}$, exchangable with $\left.\mathrm{D}_{2} \mathrm{O}\right)$; Elemental Analysis $\left(\mathrm{C}_{24} \mathrm{H}_{19} \mathrm{~N}_{4} \mathrm{O}_{3} \mathrm{Cl}_{2} \mathrm{Br}\right)$, Found\% (Calculated\%): C, 51.26 (51.27); H, 3.40 (3.41); N, 9.95 (9.96).

\section{2-(Morpholinomethylamino)-4-(7- chlorocoumarin-3-yl)-6-(3,5- dichlorophenyl)pyrimidine (15a)}

Yield: 40 \%; m.p.: $185-187{ }^{\circ} \mathrm{C}$; $\mathrm{R}_{\mathrm{f}}$ : 0.68; IR (KBr) $\mathrm{cm}^{-1}$ : $3285(\mathrm{~N}-\mathrm{H}), 1705(\mathrm{C}=\mathrm{O}), 1607(\mathrm{C}=\mathrm{N}), 1541$ $(\mathrm{C}=\mathrm{C}), 1133$ (C-O-C); 'H-NMR ( $\mathrm{CDCl}_{3}$, DMSO$\left.\mathrm{d}_{6}\right) \delta \mathrm{ppm}: 2.63\left(\mathrm{t}, \mathrm{J}=8 \mathrm{~Hz}, 4 \mathrm{H},-\mathrm{CH}_{2}-\mathrm{N}^{-} \mathrm{CH}_{2}-\right)$, $3.54\left(\mathrm{t}, \mathrm{J}=8 \mathrm{~Hz}, 4 \mathrm{H},-\mathrm{CH}_{2}-\mathrm{O}-\mathrm{CH}_{2}-\right), 4.39(\mathrm{~d}, \mathrm{~J}=$ $\left.12 \mathrm{~Hz}, 2 \mathrm{H},-\mathrm{NH}-\mathrm{CH}_{2}-\mathrm{N}-\right)$, 6.97-7.68 (m, 8H, Ar-H), $7.80\left(\mathrm{~s}, 1 \mathrm{H}, \mathrm{NH}\right.$, exchangable with $\left.\mathrm{D}_{2} \mathrm{O}\right)$; Elemental Analysis $\left(\mathrm{C}_{24} \mathrm{H}_{19} \mathrm{~N}_{4} \mathrm{O}_{3} \mathrm{Cl}_{3}\right)$, Found\% (Calculated\%): C, 55.65 (55.67); H, 3.69 (3.70); $\mathrm{N}, \quad 10.80$ (10.82); Mass (m/z): $517 \quad\left(\mathrm{M}^{+}\right.$, $\left.\mathrm{C}_{24} \mathrm{H}_{19} \mathrm{~N}_{4} \mathrm{O}_{3} \mathrm{Cl}_{3}\right), \quad 518 \quad\left(\mathrm{M}^{+}+1\right), \quad 199 \quad(100 \%$, $\mathrm{C}_{9} \mathrm{H}_{6} \mathrm{NCl}_{2}$ ).

\section{2-(Morpholinomethylamino)-4-(7- bromocoumarin-3-yl)-6-(3,5- dichlorophenyl)pyrimidine (16a)}

Yield: 55 \%; m.p.: $173-175{ }^{\circ} \mathrm{C}$; $\mathrm{R}_{\mathrm{f}}$ : 0.73; IR ( $\left.\mathrm{KBr}\right)$ $\mathrm{cm}^{-1}: 3281(\mathrm{~N}-\mathrm{H}), 1707(\mathrm{C}=\mathrm{O}), 1610(\mathrm{C}=\mathrm{N}), 1543$ $(\mathrm{C}=\mathrm{C}), 1130$ (C-O-C); ${ }^{\mathrm{H}} \mathrm{H}-\mathrm{NMR}\left(\mathrm{CDCl}_{3}\right.$, DMSO$\left.\mathrm{d}_{6}\right) \delta$ ppm: $2.64\left(\mathrm{t}, \mathrm{J}=8 \mathrm{~Hz}, 4 \mathrm{H},-\mathrm{CH}_{2}-\mathrm{N}^{-} \mathrm{CH}_{2}-\right)$, $3.51\left(\mathrm{t}, \mathrm{J}=8 \mathrm{~Hz}, 4 \mathrm{H},-\mathrm{CH}_{2}-\mathrm{O}-\mathrm{CH}_{2}-\right), 4.27(\mathrm{~d}, \mathrm{~J}=$ $\left.12 \mathrm{~Hz}, 2 \mathrm{H},-\mathrm{NH}-\mathrm{CH}_{2}-\mathrm{N}-\right)$, 6.96-7.70 (m, 8H, Ar-H), $7.83\left(\mathrm{~s}, 1 \mathrm{H}, \mathrm{NH}\right.$, exchangable with $\left.\mathrm{D}_{2} \mathrm{O}\right)$; Elemental Analysis $\left(\mathrm{C}_{24} \mathrm{H}_{19} \mathrm{~N}_{4} \mathrm{O}_{3} \mathrm{Cl}_{2} \mathrm{Br}\right)$, Found \% (Calculated \%): C, 51.26 (51.27); H, 3.40 (3.41); N, 9.95 (9.96).

\section{2-(Morpholinomethylamino)-4-(7- chlorocoumarin-3-yl)-6-(3,4- dichlorophenyl)pyrimidine (17a)}

Yield: $45 \%$; m.p.: $160-162{ }^{\circ} \mathrm{C}$; $\mathrm{R}_{\mathrm{f}}$ : 0.71; IR (KBr) $\mathrm{cm}^{-1}$ : $3286(\mathrm{~N}-\mathrm{H}), 1705(\mathrm{C}=\mathrm{O}), 1606(\mathrm{C}=\mathrm{N}), 1541$ $(\mathrm{C}=\mathrm{C}), 1132(\mathrm{C}-\mathrm{O}-\mathrm{C})$; ${ }^{1} \mathrm{H}-\mathrm{NMR}\left(\mathrm{CDCl}_{3}\right.$, DMSO$\left.\mathrm{d}_{6}\right) \delta$ ppm: $2.68\left(\mathrm{t}, \mathrm{J}=8 \mathrm{~Hz}, 4 \mathrm{H},-\mathrm{CH}_{2}-\mathrm{N}^{-} \mathrm{CH}_{2}-\right.$ ), $3.57\left(\mathrm{t}, \mathrm{J}=8 \mathrm{~Hz}, 4 \mathrm{H},-\mathrm{CH}_{2}-\mathrm{O}-\mathrm{CH}_{2}-\right), 4.33(\mathrm{~d}, \mathrm{~J}=$ $\left.12 \mathrm{~Hz}, 2 \mathrm{H},-\mathrm{NH}-\mathrm{CH}_{2}-\mathrm{N}-\right), 6.95-7.72(\mathrm{~m}, 8 \mathrm{H}, \mathrm{Ar}-\mathrm{H})$, $7.85\left(\mathrm{~s}, 1 \mathrm{H}, \mathrm{NH}\right.$, exchangable with $\left.\mathrm{D}_{2} \mathrm{O}\right)$; Elemental Analysis $\left(\mathrm{C}_{24} \mathrm{H}_{19} \mathrm{~N}_{4} \mathrm{O}_{3} \mathrm{Cl}_{3}\right)$, Found \% (Calculated \%): C, 55.66 (55.67); H, 3.69 (3.70); $\mathrm{N}, 10.81$ (10.82).

\section{2-(Morpholinomethylamino)-4-(7- bromocoumarin-3-yl)-6-(3,4- dichlorophenyl)pyrimidine (18a)}

Yield: 45 \%; m.p.: $191-193{ }^{\circ} \mathrm{C}$; $\mathrm{R}_{\mathrm{f}}$ : 0.82; IR (KBr) $\mathrm{cm}^{-1}$ : $3289(\mathrm{~N}-\mathrm{H}), 1709(\mathrm{C}=\mathrm{O}), 1605(\mathrm{C}=\mathrm{N}), 1544$
$(\mathrm{C}=\mathrm{C}), 1135$ (C-O-C); ${ }^{1} \mathrm{H}-\mathrm{NMR}\left(\mathrm{CDCl}_{3}\right.$, DMSO$\left.\mathrm{d}_{6}\right) \delta \mathrm{ppm}: 2.66\left(\mathrm{t}, \mathrm{J}=8 \mathrm{~Hz}, 4 \mathrm{H},-\mathrm{CH}_{2}-\mathrm{N}^{-} \mathrm{CH}_{2}-\right)$, $3.56\left(\mathrm{t}, \mathrm{J}=8 \mathrm{~Hz}, 4 \mathrm{H},-\mathrm{CH}_{2}-\mathrm{O}-\mathrm{CH}_{2}-\right), 4.35(\mathrm{~d}, \mathrm{~J}=$ $\left.12 \mathrm{~Hz}, 2 \mathrm{H},-\mathrm{NH}-\mathrm{CH}_{2}-\mathrm{N}-\right), 6.95-7.73(\mathrm{~m}, 8 \mathrm{H}, \mathrm{Ar}-\mathrm{H})$, $7.88\left(\mathrm{~s}, \quad 1 \mathrm{H}, \mathrm{NH}\right.$, exchangable with $\left.\mathrm{D}_{2} \mathrm{O}\right)$; Elemental Analysis $\left(\mathrm{C}_{24} \mathrm{H}_{19} \mathrm{~N}_{4} \mathrm{O}_{3} \mathrm{Cl}_{2} \mathrm{Br}\right)$, Found \% (Calculated \%): C, 51.26 (51.27); H, 3.40 (3.41); $\mathrm{N}, \quad 9.95$ (9.96); Mass (m/z): $562\left(\mathrm{M}^{+}\right.$, $\left.\mathrm{C}_{24} \mathrm{H}_{19} \mathrm{~N}_{4} \mathrm{O}_{3} \mathrm{Cl}_{2} \mathrm{Br}\right), \quad 564 \quad\left(\mathrm{M}^{+}+2\right), \quad 199 \quad(100 \%$, $\mathrm{C}_{9} \mathrm{H}_{6} \mathrm{NCl}_{2}$ ).

\section{Antimicrobial activity}

The antimicrobial activity data of the title compounds (1a-18a) at different concentrations against Gram positive bacteria, Gram negative bacteria and fungi is provided in Table 1, Table 2 and Table 3, respectively. For the explanation of the antimicrobial activity result this discussion, the zone of inhibition produced by the MIC of standard drugs, ofloxacin and ketoconazole, has been considered as $100 \%$ for comparing the antibacterial activity and antifungal activity data of the title compounds (1a-18a), respectively.

The antibacterial activity of ofloxacin against Gram positive bacteria revealed that it has a MIC value of $25 \mu \mathrm{g} / \mathrm{mL}$ against $S$. aureus, E. faecalis and $S$. epidermidis; and it has a MIC value of $12.5 \mu \mathrm{g} / \mathrm{mL}$ against $B$. subtilis and $B$. cereus. The antibacterial activity of the title compounds (1a18a) with respect to ofloxacin revealed that the compound 4a $(\mathrm{MIC}=100 \mu \mathrm{g} / \mathrm{mL})$ displayed highest activity of about $93.98 \%$ with $p<0.05$ against $S$. aureus; the compound 15a $(\mathrm{MIC}=$ $125 \mu \mathrm{g} / \mathrm{mL}$ ) displayed highest activity of about $83.60 \%$ with $p<0.0001$ against $E$. faecalis; the compound 9a (MIC $=100 \mu \mathrm{g} / \mathrm{mL})$ displayed highest activity of about $91.03 \%(p<0.0001)$ against $S$. epidermidis; the compound 14a (MIC $=150 \mu \mathrm{g} / \mathrm{mL}$ ) displayed highest activity of about $88.5 \%$ with $p<0.0001$ against $B$. subtilis; and the compound 18a $(\mathrm{MIC}=100 \mu \mathrm{g} / \mathrm{mL})$ displayed highest activity of about $91.45 \%$ with $p<0.0001$ against $B$. cereus.

Some compounds exhibited good but statistically non-significant antibacterial activity results $(p>$ 0.05 ) with respect to ofloxacin, for example, the compound 13a (MIC $=100 \mu \mathrm{g} / \mathrm{mL})$ displayed about $99.88 \%$ activity against $S$. aureus; compound 18a (MIC $=125 \mu \mathrm{g} / \mathrm{mL})$ displayed about $94.75 \%$ activity against $S$. aureus; and compounds 15a $(\mathrm{MIC}=175 \mu \mathrm{g} / \mathrm{mL})$ displayed about $98 \%$ activity against $S$. epidermidis. The antibacterial activity produced by the compounds 4a, 9a, 13a, 14a, 15a and 18a was lower than the antibacterial activity produced by ofloxacin 
Table 1: Antibacterial activity data of the title compounds (1a-18a) against Gram positive bacteria

\begin{tabular}{|c|c|c|c|c|c|}
\hline \multirow[t]{2}{*}{ Compound } & \multicolumn{5}{|c|}{ Zone of inhibition ( $\mathrm{mm} \pm \mathrm{SD}$ ) and corresponding MIC $(\mu \mathrm{g} / \mathrm{mL})$ value in bracket } \\
\hline & S. aureus & E. faecalis & S. epidermidis & B. subtilis & B. cereus \\
\hline $1 \mathbf{a}$ & $12.28 \pm 0.27^{a}(50)$ & $17.12 \pm 0.26^{a}(50)$ & $16.82 \pm 0.26^{a}(75)$ & $10.50 \pm 0.30^{a}(50)$ & $18.77 \pm 0.24^{a}(100)$ \\
\hline $2 a$ & $10.73 \pm 0.23^{a}(75)$ & $20.67 \pm 0.29^{a}(100)$ & $9.20 \pm 0.23^{a}(200)$ & $16.53 \pm 0.25^{a}(50)$ & $13.97 \pm 0.28^{a}(150)$ \\
\hline $3 \mathbf{a}$ & $17.02 \pm 0.32^{a}(100)$ & $13.53 \pm 0.33^{a}(150)$ & $16.55 \pm 0.29^{a}(100)$ & $18.07 \pm 0.31^{a}(125)$ & $20.28 \pm 0.27^{a}(125)$ \\
\hline $4 a$ & $24.20 \pm 0.30^{c}(100)$ & $20.38 \pm 0.30^{a}(125)$ & $24.08 \pm 0.26^{a}(75)$ & $21.05 \pm 0.31^{a}(100)$ & $24.78 \pm 0.32^{a}(100)$ \\
\hline $5 \mathbf{a}$ & $13.27 \pm 0.28^{c}(50)$ & $11.62 \pm 0.28^{a}(75)$ & $19.72 \pm 0.33^{a}(75)$ & $14.62 \pm 0.42^{a}(25)$ & $19.92 \pm 0.28^{a}(50)$ \\
\hline $6 \mathbf{a}$ & $5.27 \pm 0.26^{a}(200)$ & $17.48 \pm 0.28^{a}(50)$ & $16.12 \pm 0.36^{a}(150)$ & $22.05 \pm 0.29^{a}(150)$ & $13.95 \pm 0.24^{a}(50)$ \\
\hline $7 \mathbf{a}$ & $11.08 \pm 0.33^{a}(150)$ & $14.02 \pm 0.16^{a}(75)$ & $19.20 \pm 0.32^{a}(100)$ & $15.05 \pm 0.24^{a}(75)$ & $22.97 \pm 0.22^{a}(150)$ \\
\hline $8 \mathbf{a}$ & $20.22 \pm 0.25^{a}(100)$ & $16.42 \pm 0.27^{a}(150)$ & $11.63 \pm 0.51^{a}(50)$ & $15.27 \pm 0.36^{a}(50)$ & $21.38 \pm 0.39^{a}(75)$ \\
\hline $9 a$ & $21.62 \pm 0.41^{a}(75)$ & $23.22 \pm 0.37^{a}(50)$ & $25.08 \pm 0.35^{a}(100)$ & $19.97 \pm 0.33^{a}(100)$ & $21.15 \pm 0.40^{a}(100)$ \\
\hline $10 a$ & $14.80 \pm 0.42^{a}(50)$ & $12.25 \pm 0.34^{a}(100)$ & $18.65 \pm 0.35^{a}(50)$ & $19.50 \pm 0.31^{a}(125)$ & $9.23 \pm 0.39^{a}(175)$ \\
\hline $11 a$ & $9.72 \pm 0.43^{a}(125)$ & $16.65 \pm 0.38^{a}(75)$ & $14.03 \pm 0.31^{a}(50)$ & $12.07 \pm 0.36^{a}(50)$ & $20.02 \pm 0.33^{a}(100)$ \\
\hline $12 a$ & $20.62 \pm 0.33^{a}(100)$ & $11.80 \pm 0.37^{a}(175)$ & $9.28 \pm 0.40^{a}(175)$ & $17.02 \pm 0.42^{a}(150)$ & $23.57 \pm 0.41^{a}(125)$ \\
\hline $13 a$ & $25.72 \pm 0.36^{a}(100)$ & $21.13 \pm 0.36^{a}(150)$ & $19.30 \pm 0.38^{a}(50)$ & $25.17 \pm 0.45^{a}(100)$ & $25.65 \pm 0.30^{a}(100)$ \\
\hline $14 a$ & $21.87 \pm 0.43^{a}(125)$ & $18.83 \pm 0.41^{a}(100)$ & $24.02 \pm 0.26^{a}(100)$ & $27.95 \pm 0.29^{a}(150)$ & $22.80 \pm 0.34^{a}(100)$ \\
\hline $15 a$ & $20.20 \pm 0.35^{a}(100)$ & $24.02 \pm 0.39^{a}(125)$ & $27.00 \pm 0.26^{a}(175)$ & $16.77 \pm 0.40^{a}(100)$ & $19.15 \pm 0.41^{a}(75)$ \\
\hline $16 \mathbf{a}$ & $14.98 \pm 0.37^{a}(50)$ & $18.50 \pm 0.25^{a}(75)$ & $12.08 \pm 0.47^{a}(50)$ & $8.48 \pm 0.41^{a}(175)$ & $18.20 \pm 0.35^{a}(150)$ \\
\hline $17 a$ & $18.95 \pm 0.28^{a}(75)$ & $13.98 \pm 0.27^{a}(150)$ & $21.03 \pm 0.27^{a}(75)$ & $15.38 \pm 0.37^{a}(50)$ & $24.27 \pm 0.31^{a}(125)$ \\
\hline $18 a$ & $24.40 \pm 0.36^{a}(125)$ & $21.53 \pm 0.38^{a}(100)$ & $24.05 \pm 0.28^{a}(150)$ & $4.63 \pm 0.39^{a}(200)$ & $26.43 \pm 0.36^{a}(100)$ \\
\hline Ofloxacin & $25.75 \pm 0.43^{a}(25)^{\prime}$ & $28.73 \pm 0.43^{a}(25)$ & $27.55 \pm 0.41^{a}(25)^{\prime}$ & $31.58 \pm 0.41^{a}(12.5)$ & $28.90 \pm 0.27^{a}(12.5)$ \\
\hline Negative & $0.0 \pm 0.0$ & $0.0 \pm 0.0$ & $0.0 \pm 0.0$ & $0.0 \pm 0.0$ & $0.0 \pm 0.0$ \\
\hline
\end{tabular}

Values in parenthesis represent the corresponding MIC $(\mu \mathrm{g} / \mathrm{mL}) ;{ }^{a} p<0.0001,{ }^{b} p<0.001,{ }^{c} p<0.05,,{ }^{d} p>0.05$ 
Table 2: Antibacterial activity data of the title compounds (1a-18a) against Gram negative bacteria

\begin{tabular}{|c|c|c|c|c|c|}
\hline \multirow[t]{2}{*}{ Compound } & \multicolumn{5}{|c|}{ Zone of inhibition ( $\mathrm{mm} \pm \mathrm{SD}$ and corresponding MIC $(\mu \mathrm{g} / \mathrm{mL})$} \\
\hline & E. coli & P. aeruginosa & K. pneumonia & B. bronchiseptica & P. vulgaris \\
\hline 1a & $16.42 \pm 0.42^{a}(100)$ & $19.73 \pm 0.24^{a}(100)$ & $21.73 \pm 0.43^{a}(75)$ & $23.78 \pm 0.38^{a}(100)$ & $12.78 \pm 0.27^{a}(200)$ \\
\hline $2 a$ & $13.05 \pm 0.28^{a}(150)$ & $14.72 \pm 0.27^{a}(150)$ & $19.70 \pm 0.26^{a}(125)$ & $16.67 \pm 0.29^{a}(50)$ & $14.07 \pm 0.27^{a}(50)$ \\
\hline $3 a$ & $21.47 \pm 0.35^{a}(75)$ & $20.47 \pm 0.31^{a}(75)$ & $27.67 \pm 0.25^{a}(100)$ & $25.52 \pm 0.30^{a}(125)$ & $16.42 \pm 0.25^{a}(50)$ \\
\hline $4 \mathbf{a}$ & $25.87 \pm 0.27^{a}(75)$ & $27.45 \pm 0.29^{a}(50)$ & $21.67 \pm 0.33^{a}(75)$ & $23.22 \pm 0.27^{a}(100)$ & $23.30 \pm 0.41^{a}(100)$ \\
\hline $5 a$ & $13.70 \pm 0.39^{a}(175)$ & $11.92 \pm 0.32^{a}(200)$ & $19.43 \pm 0.41^{a}(50)$ & $17.20 \pm 0.39^{a}(150)$ & $8.78 \pm 0.38^{a}(175)$ \\
\hline $6 a$ & $18.92 \pm 0.42^{a}(100)$ & $21.60 \pm 0.39^{a}(75)$ & $16.98 \pm 0.32^{a}(50)$ & $11.32 \pm 0.41^{a}(200)$ & $15.97 \pm 0.37^{a}(75)$ \\
\hline $7 a$ & $7.48 \pm 0.41^{a}(200)$ & $15.27 \pm 0.35^{a}(150)$ & $11.67 \pm 0.35^{a}(150)$ & $18.47 \pm 0.34^{a}(75)$ & $12.53 \pm 0.33^{a}(150)$ \\
\hline $8 \mathbf{a}$ & $16.22 \pm 0.36^{a}(50)$ & $20.12 \pm 0.35^{a}(100)$ & $12.40 \pm 0.41^{a}(125)$ & $13.95 \pm 0.34^{a}(175)$ & $21.88 \pm 0.36^{a}(100)$ \\
\hline $9 a$ & $21.25 \pm 0.30^{a}(100)$ & $27.53 \pm 0.37^{a}(100)$ & $23.20 \pm 0.35^{a}(100)$ & $24.05 \pm 0.30^{a}(100)$ & $27.80 \pm 0.26^{a}(75)$ \\
\hline $10 a$ & $24.03 \pm 0.28^{a}(100)$ & $21.52 \pm 0.27^{a}(50)$ & $20.25 \pm 0.38^{a}(100)$ & $27.63 \pm 0.29^{a}(100)$ & $24.25 \pm 0.38^{a}(100)$ \\
\hline $11 a$ & $13.83 \pm 0.25^{a}(150)$ & $11.60 \pm 0.45^{a}(75)$ & $13.25 \pm 2.38^{a}(175)$ & $20.08 \pm 0.39^{a}(150)$ & $17.37 \pm 0.45^{a}(50)$ \\
\hline $12 a$ & $9.70 \pm 0.24^{a}(200)$ & $14.53 \pm 0.35^{a}(50)$ & $8.47 \pm 0.38^{a}(75)$ & $15.73 \pm 0.29^{a}(150)$ & $14.05 \pm 0.38^{a}(50)$ \\
\hline $13 a$ & $16.42 \pm 0.30^{a}(50)$ & $24.18 \pm 0.37^{a}(75)$ & $26.40 \pm 0.39^{a}(75)$ & $20.38 \pm 0.32^{a}(125)$ & $22.27 \pm 0.33^{a}(75)$ \\
\hline $14 a$ & $16.45 \pm 0.45^{a}(50)$ & $18.33 \pm 0.40^{a}(75)$ & $22.63 \pm 0.39^{a}(100)$ & $12.45 \pm 0.51^{a}(50)$ & $12.83 \pm 0.34^{a}(125)$ \\
\hline $15 a$ & $14.62 \pm 0.28^{a}(75)$ & $21.63 \pm 0.38^{a}(100)$ & $16.98 \pm 0.28^{a}(50)$ & $24.85 \pm 0.17^{a}(75)$ & $21.20 \pm 0.31^{a}(100)$ \\
\hline $16 a$ & $20.12 \pm 0.34^{a}(100)$ & $24.77 \pm 0.31^{a}(75)$ & $21.48 \pm 0.29^{a}(100)$ & $24.10 \pm 0.37^{a}(100)$ & $19.08 \pm 0.33^{a}(100)$ \\
\hline $17 a$ & $12.30 \pm 0.40^{a}(125)$ & $9.37 \pm 0.41^{a}(150)$ & $11.25 \pm 0.38^{a}(175)$ & $18.27 \pm 0.38^{a}(125)$ & $13.83 \pm 0.25^{a}(150)$ \\
\hline $18 a$ & $22.75 \pm 0.42^{a}(75)$ & $26.37 \pm 0.37^{a}(100)$ & $28.72 \pm 0.29^{c}(100)$ & $24.32 \pm 0.43^{a}(100)$ & $22.95 \pm 0.36^{a}(100)$ \\
\hline Ofloxacin & $28.48 \pm 0.37^{a}(12.5)$ & $31.12 \pm 0.14^{a}(12.5)$ & $31.55 \pm 0.19^{a}(12.5)$ & $32.22 \pm 0.20^{a}(25)$ & $29.98 \pm 0.29^{a}(12.5)$ \\
\hline $\begin{array}{l}\text { Negative } \\
\text { Control }\end{array}$ & $0.0 \pm 0.0$ & $0.0 \pm 0.0$ & $0.0 \pm 0.0$ & $0.0 \pm 0.0$ & $0.0 \pm 0.0$ \\
\hline
\end{tabular}

Values in parenthesis represent the corresponding MIC $(\mu \mathrm{g} / \mathrm{mL}) ;{ }^{a} p<0.0001,{ }^{b} p<0.001,{ }^{c} p<0.05,{ }^{d} p>0.05$ 
Table 3: Antifungal activity data of the title compounds (1a-18a) against fung

\begin{tabular}{|c|c|c|c|c|c|}
\hline \multirow[t]{2}{*}{ Compound } & \multicolumn{5}{|c|}{ Zone of inhibition $(\mathrm{mm} \pm \mathrm{SD}$ and corresponding MIC $(\mu \mathrm{g} / \mathrm{mL})$} \\
\hline & C. albicans & A. niger & A. flavus & M. purpureous & P. citrinum \\
\hline 19 & $18.88 \pm 0.33^{a}(75)$ & $21.52 \pm 0.31^{a}(75)$ & $17.30 \pm 0.23^{a}(50)$ & $15.05 \pm 0.38^{a}(50)$ & $20.38 \pm 0.41^{a}(100)$ \\
\hline $2 a$ & $22.77 \pm 0.39^{a}(100)$ & $14.50 \pm 0.34^{a}(50)$ & $19.45 \pm 0.34^{a}(50)$ & $18.43 \pm 0.35^{a}(50)$ & $19.18 \pm 0.40^{a}(50)$ \\
\hline $3 a$ & $22.58 \pm 0.32^{a}(100)$ & $21.95 \pm 0.39^{a}(75)$ & $25.90 \pm 0.33^{a}(75)$ & $24.47 \pm 0.36^{a}(100)$ & $28.13 \pm 0.29^{a}(75)$ \\
\hline $4 a$ & $19.37 \pm 0.44^{a}(50)$ & $20.28 \pm 0.40^{a}(100)$ & $17.53 \pm 0.35^{a}(50)$ & $18.27 \pm 0.38^{a}(50)$ & $21.53 \pm 0.47^{a}(75)$ \\
\hline $5 \mathbf{a}$ & $24.17 \pm 0.37^{a}(75)$ & $27.08 \pm 0.26^{c}(100)$ & $20.45 \pm 0.50^{a}(50)$ & $27.70 \pm 0.16^{a}(50)$ & $23.35 \pm 0.38^{a}(100)$ \\
\hline $6 a$ & $20.67 \pm 0.52^{a}(50)$ & $16.92 \pm 0.55^{a}(50)$ & $23.15 \pm 0.34^{a}(125)$ & $20.30 \pm 0.35^{a}(100)$ & $22.13 \pm 0.32^{a}(100)$ \\
\hline $7 a$ & $23.18 \pm 0.34^{a}(50)$ & $22.53 \pm 0.36^{a}(75)$ & $26.20 \pm 0.27^{a}(75)$ & $27.10 \pm 0.43^{a}(75)$ & $24.87 \pm 0.60^{a}(100)$ \\
\hline $8 a$ & $17.25 \pm 0.38^{a}(125)$ & $24.42 \pm 0.40^{a}(75)$ & $17.97 \pm 3.09^{a}(50)$ & $16.97 \pm 0.48^{a}(50)$ & $16.67 \pm 0.41^{a}(50)$ \\
\hline $9 a$ & $25.28 \pm 0.37^{a}(75)$ & $27.50 \pm 0.20^{a}(100)$ & $25.53 \pm 0.30^{a}(75)$ & $21.27 \pm 0.35^{a}(100)$ & $19.87 \pm 0.34^{a}(125)$ \\
\hline $10 a$ & $27.78 \pm 0.44^{a}(100)$ & $26.32 \pm 0.39^{a}(75)$ & $24.85 \pm 0.45^{a}(125)$ & $27.83 \pm 0.32^{a}(100)$ & $27.18 \pm 0.28^{a}(50)$ \\
\hline $11 a$ & $24.62 \pm 0.45^{a}(75)$ & $22.73 \pm 0.42^{a}(75)$ & $26.62 \pm 0.43^{a}(100)$ & $20.75 \pm 0.36^{a}(75)$ & $24.32 \pm 0.33^{c}(125)$ \\
\hline $12 a$ & $22.20 \pm 0.35^{a}(50)$ & $18.80 \pm 0.50^{a}(50)$ & $17.33 \pm 0.40^{a}(100)$ & $22.40 \pm 0.41^{a}(50)$ & $19.28 \pm 0.34^{a}(50)$ \\
\hline $13 a$ & $16.65 \pm 0.40^{a}(125)$ & $23.15 \pm 0.30^{a}(75)$ & $18.22 \pm 0.38^{a}(50)$ & $14.07 \pm 0.38^{a}(100)$ & $23.82 \pm 0.27^{a}(100)$ \\
\hline $14 a$ & $14.13 \pm 0.28^{a}(150)$ & $19.78 \pm 0.43^{a}(50)$ & $23.97 \pm 0.25^{b}(50)$ & $18.98 \pm 0.32^{a}(75)$ & $21.60 \pm 0.32^{a}(75)$ \\
\hline $15 a$ & $25.60 \pm 0.32^{a}(125)$ & $22.22 \pm 0.35^{a}(75)$ & $20.53 \pm 0.44^{a}(50)$ & $26.92 \pm 0.28^{a}(100)$ & $28.17 \pm 0.27^{b}(125)$ \\
\hline $16 a$ & $24.50 \pm 0.34^{a}(75)$ & $27.03 \pm 0.27^{c}(100)$ & $26.90 \pm 0.34^{a}(75)$ & $22.03 \pm 0.41^{a}(125)$ & $18.10 \pm 0.39^{a}(50)$ \\
\hline $17 a$ & $26.32 \pm 0.36^{a}(100)$ & $27.28 \pm 0.25^{a}(100)$ & $24.22 \pm 0.29^{c}(125)$ & $19.60 \pm 0.42^{a}(50)$ & $20.32 \pm 0.45^{a}(100)$ \\
\hline $18 a$ & $18.27 \pm 0.34^{a}(100)$ & $17.83 \pm 0.46^{a}(50)$ & $21.17 \pm 0.34^{a}(50)$ & $24.23 \pm 0.25^{a}(75)$ & $15.78 \pm 0.28^{a}(50)$ \\
\hline Ketoconazole & $32.30 \pm 0.29^{a}(12.5)$ & $28.70 \pm 0.38^{a}(12.5)$ & $27.97 \pm 0.52^{a}(25)$ & $31.90 \pm 0.34^{a}(12.5)$ & $26.07 \pm 0.28^{a}(25)$ \\
\hline $\begin{array}{l}\text { Negative } \\
\text { Control }\end{array}$ & $0.0 \pm 0.0$ & $0.0 \pm 0.0$ & $0.0 \pm 0.0$ & $0.0 \pm 0.0$ & $0.0 \pm 0.0$ \\
\hline
\end{tabular}

Values in parenthesis represent the corresponding MIC $(\mu \mathrm{g} / \mathrm{mL}) ;{ }^{a} p<0.0001,{ }^{b} p<0.001,{ }^{c} p<0.05$ 
and they also had a higher MIC values than ofloxacin. Other compounds did not produced comparable antibacterial activity against Gram positive bacteria even at higher concentrations with respect to ofloxacin.

The antibacterial activity of ofloxacin against Gram negative bacteria revealed that it has a MIC value of $12.5 \mu \mathrm{g} / \mathrm{mL}$ against $E$. coli; $P$. aeruginosa, K. pneumonia and $P$. vulgaris; and it has a MIC value of $25 \mu \mathrm{g} / \mathrm{mL}$ against $B$. bronchiseptica. The antibacterial activity of the title compounds (1a-18a) with respect to ofloxacin revealed that the compound $\mathbf{4 a}$ (MIC = $75 \mu \mathrm{g} / \mathrm{mL}$ ) displayed the highest activity of about $90.83 \%$ with $p<0.0001$ against $E$. coli; the compounds $4 \mathrm{a}(\mathrm{MIC}=50 \mu \mathrm{g} / \mathrm{mL})$ and $9 \mathrm{a}(\mathrm{MIC}=$ $100 \mu \mathrm{g} / \mathrm{mL}$ ) displayed highest activity of about $88.20 \%$ and $88.46 \%$, respectively, with $p<$ 0.0001 against $P$. aeruginosa; the compound 18a $(\mathrm{MIC}=100 \mu \mathrm{g} / \mathrm{mL})$ displayed highest activity of about $91.03 \%$ with $p<0.05$ against $K$. pneumonia; the compound 10a (MIC $=100$ $\mu \mathrm{g} / \mathrm{mL}$ ) displayed highest activity of about 85.75 $\%(p<0.0001)$; and the compound 9a (MIC = 75 $\mu \mathrm{g} / \mathrm{mL}$ ) displayed highest activity of about 92.72 $\%(p<0.0001)$ against $P$. vulgaris. The antibacterial activity produced by the compounds $4 a, 9 a, 10 a$ and $18 a$ was lower than the antibacterial activity produced by ofloxacin and they also had a higher MIC value than ofloxacin. Other compounds did not produced comparable antibacterial activity against Gram negative bacteria even at higher concentrations with respect to ofloxacin.

The antifungal activity of ketoconazole against fungi revealed that it has a MIC value of 12.5 $\mu \mathrm{g} / \mathrm{mL}$ against $C$. albicans, $A$. niger and $M$. purpureous; and it has a MIC value of $25 \mu \mathrm{g} / \mathrm{mL}$ against $A$. flavus and $P$. citrinum. The antifungal activity of the title compounds (1a-18a) with respect to ketoconazole revealed that the compound 10a $(\mathrm{MIC}=100 \mu \mathrm{g} / \mathrm{mL})$ displayed highest activity of about $86 \%$ with $p<0.0001$ against $C$. albicans; the compounds $5 \mathrm{a}$ (MIC $=$ $100 \mu \mathrm{g} / \mathrm{mL}$ ) and 16a (MIC $=100 \mu \mathrm{g} / \mathrm{mL}$ ) displayed highest activity of about $94.35 \%$ and 94.18, respectively, with $p<0.05$ against $A$. niger, the compound 17a $(\mathrm{MIC}=125 \mu \mathrm{g} / \mathrm{mL}$ ) displayed highest activity of about $86.59 \%$ with $p$ $<0.05$ against $A$. flavus; the compounds 5a (MIC $=50 \mu \mathrm{g} / \mathrm{mL}), 7 \mathrm{a}(\mathrm{MIC}=75 \mu \mathrm{g} / \mathrm{mL})$, and 10a (MIC $=100 \mu \mathrm{g} / \mathrm{mL}$ ) displayed highest activity of about $86.83,84.95$, and $87.24 \%$, respectively, with $p<$ 0.0001 against $M$. purpureous; and the compounds 3a (MIC $=75 \mu \mathrm{g} / \mathrm{mL}$ ) and 15a (MIC $=125 \mu \mathrm{g} / \mathrm{mL}$ ) displayed highest activity of about
107.90 and $108.05 \%$, respectively, against $P$. citrinum. The antifungal activity produced by the compounds $3 \mathrm{a}$ and $15 \mathrm{a}$ was more than ketoconazole and statistically significant also. However, their MIC was also high. Some compounds exhibited good but statistically nonsignificant antifungal activity $(p>0.05)$ with respect to ketoconazole, for example, the compound 9a $(\mathrm{MIC}=100 \mu \mathrm{g} / \mathrm{mL})$ and compound 17a $(\mathrm{MIC}=100 \mu \mathrm{g} / \mathrm{mL})$, respectively, displayed about 95.81 and $95.05 \%$ activity against $A$. niger; the compounds $3 \mathrm{a}(\mathrm{MIC}=75 \mu \mathrm{g} / \mathrm{mL}), 7 a$ $(\mathrm{MIC}=75 \mu \mathrm{g} / \mathrm{mL}), 9 \mathrm{a}(\mathrm{MIC}=75 \mu \mathrm{g} / \mathrm{mL}), 11 \mathrm{a}$ $(\mathrm{MIC}=100 \mu \mathrm{g} / \mathrm{mL})$, and 16a $(\mathrm{MIC}=75 \mu \mathrm{g} / \mathrm{mL})$, respectively, displayed activity of about 92.59, 93.67, 91.27\%, 97.17, and $96.17 \%$ against $A$. flavus. The antifungal activity produced by the compounds 3a, 5a, 7a, 9a, 10a, 11a, 16a, and 17a was lower than the antifungal activity produced by ketoconazole and they also had a higher MIC value than ketoconazole. Other compounds did not produced comparable antifungal activity against fungi even at higher concentrations with respect to ketoconazole.

\section{DISCUSSION}

A total of eighteen new compounds (1a-18a) were synthesized, and their structures were confirmed on the basis of their IR, ${ }^{1} \mathrm{H}-\mathrm{NMR}$, Mass and elemental analysis data. The characteristic peaks in ${ }^{1} \mathrm{H}-\mathrm{NMR}$ spectra that confirmed the formation of the compounds (1a18a) from the compounds (1-18) [25] were the appearance of the signals at $\delta(\mathrm{ppm})$ values from 2.63 to 2.72 for the four protons of $-\mathrm{CH}_{2}-\mathrm{N}^{-} \mathrm{CH}_{2}-$ portion of the morpholine moiety; from 3.50 to 3.58 for the four protons of $-\mathrm{CH}_{2}-\mathrm{O}-\mathrm{CH}_{2}$ - portion of the morpholin moiety; and from 4.27 to 4.42 for the two methylene protons of $-\mathrm{NH}^{-} \mathrm{CH}_{2}$ moiety. The compounds (1a-18a) were tested for their in vitro antimicrobial activity by serial plate dilution method [27,28] against five Grampositive bacteria; five Gram-negative bacteria; and five fungi.

Compound 3a (MIC $=75 \mu \mathrm{g} / \mathrm{mL} ; p<0.0001)$ and 15a $(\mathrm{MIC}=125 \mu \mathrm{g} / \mathrm{mL} ; p<0.001)$ produced superior antifungal activity than the standard drug, ketoconazole $(\mathrm{MIC}=25 \mu \mathrm{g} / \mathrm{mL} ; p<$ 0.0001 ) against $P$. citrinum. Compound 4 a (MIC $=100 \mu \mathrm{g} / \mathrm{mL} ; p<0.05$ ) displayed highest but moderate activity against $S$. aureus with respect to the standard drug, ofloxacin (MIC $=25 \mu \mathrm{g} / \mathrm{mL}$; $p<0.0001$ ). Compound 4a (MIC $=75 \mu \mathrm{g} / \mathrm{mL} ; p<$ 0.0001 ) also exhibited highest but moderate activity against $E$. coli with respect to the standard drug, ofloxacin (MIC $=12.5 \mu \mathrm{g} / \mathrm{mL} ; p<$ 
0.0001). Further, compound 4a showed less activity than ofloxacin and also had higher MIC values than the standard drug ofloxacin. It is evident from the antimicrobial activity data mentioned in Table 1, Table 2, and Table 3 that the title compounds are better antifungal agents than antibacterial agents. This is contrary to our earlier reported work [25]. This shows that the addition of the morpholine moiety to these type of compounds [25] increases their antifungal activity. Accordingly, there is a possibility that the replacement of the morpholine moiety by its bioisosteres, for example piperidine moiety, in these compounds may also produce promising antifungal compounds. Accordingly, this study may be extended to acquire more information about the structure activity relationships of these type of compounds. It is also believed that the synthesized compounds might be inhibiting the growth of all tested microorganism by same mechanism as earlier reported pyrimidine moiety containing drugs [15].

The structure activity relationship study of the title compounds (1a-18a) revealed that the chloro substitution with respect to positions 2,3 and 5 of the phenyl ring along with a chloro/bromo substituted coumarin moiety are critical for activity against Gram positive bacteria; the chlorosubstitution with respect to positions 2,3 , and 4 of the phenyl ring along with a chloro/bromo substituted coumarin moiety are critical for activity against Gram negative bacteria; the chlorosubstitution with respect to positions 2,4 , and 6 of the phenyl ring along with a chloro substituted coumarin moiety are critical for activity against fungi; the chloro substitution at position 2 of the phenyl ring along with a chloro/bromo substituted coumarin moiety is relatively more critical for activity against Gram positive bacteria, Gram negative bacteria and fungi.

\section{CONCLUSION}

It is evident from the antimicrobial activity data of the title compounds (1a-18a) that the compounds 3a and 15a produced higher antifungal activity than standard drug ketoconazole against $P$. citrinum. However, these compounds produced superior effect at higher concentration, and therefore, are considered to be less potent than ketoconazole. Some of the compounds displayed promising antibacterial activity at higher concentrations. It is also evident that the title compounds are better antifungal agents than as antibacterial agents. These compounds may be modified to achieve more potent antimicrobial activity. Accordingly, further studies to acquire more information about structure activity relationships are in progress in our laboratory.

\section{ACKNOWLEDGEMENT}

The authors are thankful to Central Drug Research Institute (CDRI) for generating the spectral data of the title compounds, and also to Majeedia Hospital of Jamia Hamdard for providing facilities to carry out the antimicrobial studies.

\section{CONFLICT OF INTEREST}

No conflict of interest associated with this work.

\section{CONTRIBUTION OF AUTHORS}

Mohd Imran and Abida conceived and designed the study. Abida and Abdulkhaliq J Alsalman performed the practical work. Mohd Imran and Abdulkhaliq $\mathrm{J}$ Alsalman analysed the data generated during this work. Mohd Imran and Abida wrote the manuscript which was also reviewed by Abdulkhaliq J Alsalman.

\section{REFERENCES}

1. Abida, Imran M, Hagga MAM, El-Feky SA. New chemical entities of future for infectious diseases. Eur $J$ Exp Biol 2014; 4(1): 613-624.

2. Jain KS, Chitre TS, Miniyar PB, Kathiravan MK, Bendre VS, Veer VS, Shahane SR, Shishoo CJ. Biological and medicinal significance of pyrimidines. Curr Sci 2006; 90(6): 793-703.

3. Jitendra KG, Pramod KS, Rupesh D, Anshu C, Avnesh S, Verma PK, Sambhu CM, Rakesh $K Y$, Shivjee $K$. Analgesic study of novel pyrimidine derivatives linked with coumarin moiety. Med Chem Res 2012; 21(8): 1625-1632.

4. Anshu C, Pramod KS, Prabhakar V, Nitin K, Rupesh D. Microwave assisted synthesis of novel pyrimidine derivatives and investigation of their analgesic and ulcerogenic activity. Med Chem Res 2012; 21(11): 36293645.

5. Kumar SM, Pavani M, Bhalgat CM, Deepthi R, Mounika A, Mudshinge SR, Reas IJ, Ghomi JS, Ghasemzadeh MA. Novel pyrimidine and its triazole fused derivatives: synthesis and investigation of antioxidant and antiinflammatory activity. J Serb Chem Soc 2011; 76: 679684.

6. Abu-Hashem AA, Youssef MM, Hoda AR. Synthesis, antioxidant, antituomer activities of some new thiazolopyrimidines, pyrrolothiazolopyrimidines and triazolopyrrolo thiazolopyrimidines derivatives. J Chi Chem Soc 2011; 58: 41-48.

7. Gressler V, Moura S, Flores AFC, Flores DC, Colepicolo $P$, Pinto E. Antioxidant and antimicrobial properties of 2- 
(4,5-dihydro-1H-pyrazol-1-yl)-pyrimidine and 1carboxamidino-1H-pyrazole derivatives. I Braz Chem Soc 2010; 21: 1-7.

8. Aggarwal R, Masan E, Kaushik P, Kaushik D, Sharma $C$, Aneja KR. Synthesis and biological evaluation of 7 trifluoromethylpyrazolo\{1,5-a\}pyrimidines as antiinflammatory and antimicrobial agents. J Fluorine Chem 2014; 168: 16-24.

9. Kumar N, Chauhan A, Drabu S. Synthesis of cyanopyridine and pyrimidine analogues as new antiinflammatory and antimicrobial agents. Biomed Pharmacother 2011; 65(5): 375-380.

10. Mohamed MS, Youns MM, Ahmed NM. Novel indolylpyrimidine derivatives: synthesis, antimicrobial, and antioxidant evaluations. Med Chem Res 2014; 23(7): 3374-3388.

11. Kotaiah Y, Nagaraju K, Harikrishna N, Rao CV, Yamini L, Vijjulatha M. Synthesis, docking and evaluation of antioxidant and antimicrobial activities of novel 1,2,4triazolo\{3,4-b\}\{1,3,4\}thiadiazol-6-yl)selenopheno\{2,3d\}pyrimidines. Eur J Med Chem 2014; 75: 195-102.

12. Mosaad SM, Mahmoud MY, Naglaa MA. Novel indolylpyrimidine derivatives: synthesis, antimicrobial, and antioxidant evaluations. Med Chem Res 2014; 23: 33743388.

13. Saundane AR, Yarlakatti M, Kalpana R. Synthesis, antimicrobial, and antioxidant activities of some new indole analogues containing pyrimidine and fused pyrimidine systems. Med Chem Res 2012; 21: 38093817.

14. Selvam TP, James CR, Dniandev PV, Valzita SK. A mini review of pyrimidine and fused pyrimidine marketed Drugs. Res Pharm 2012; 2(4): 1-9.

15. Sharma V, Chitranshi N, Agarwal AK. Significance and biological importance of pyrimidine in the microbial world. Int J Med Chem 2014; doi:10.1155/2014/202784.

16. Stevens DL, Dotter B, Madaras-Kelly K. A review of linezolid: the first oxazolidinone antibiotic. Expert Rev Anti Infect Ther 2004; 2(1): 51-59.

17. Aridoss G, Balasubramanian GAS, Parthiban $P$, Kabilan $S$. Synthesis, stereochemistry and antimicrobial evaluation of some $N$-morpholinoacetyl-2,6diarylpiperidin-4-ones. Eur J Med Chem 2007; 42: 851860.
18. Panneerselvam $P, \quad$ Nair RR, Vijayalakshimi G, Subramanian EH, Sridhar SK. Synthesis of Schiff bases of 4-(4-aminophenyl)-morpholine as potential antimicrobial agents. Eur J Med Chem 2005; 40: 225229.

19. Raparti V, Chitre T, Bothara K, Kumar V, Dangre S, Khachane C, Gore S, Deshmane B. Novel 4-(morpholin4-yl)-NO-(arylidene)benzohydrazides: synthesis, antimycobacterial activity and QSAR investigations. Eur J Med Chem 2009; 44: 3954-3960.

20. Wyrzykiewicz E, Wendzonka M, Kedzi B. Synthesis and antimicrobial activity of new (E)-4-[piperidino (40methylpiperidino-, morpholino-) $\mathrm{N}$-alkoxy]stilbenes. Eur J Med Chem 2006; 41: 519-525.

21. Bektas H, Ceylan S, Demirbas N, Alpay-Karaoglu S, Sokmen BB. Antimicrobial and antiurease activities of newly synthesized morpholine derivatives containing an azole nucleus. Med Chem Res 2013; 22(8): 3629-3639.

22. Nikitakis A, Kourounakis A. QSAR of substituted morpholines with antioxidant and squalene synthase inhibitory activity. Med Chem Res 2011; 20(5): 566-575.

23. Imran M, Khan SA. Synthesis of novel 3,5-disubstituted isoxazolines as potential antibacterial and antifungal agents. Indian J Heterocycl Chem 2004; 13: 213-216.

24. Khan SA, Siddiqui N, Imran M, Haque SW. Synthesis and antimicrobial screening of novel mannich bases of isatin derivative. Indian J Pharm Sci 2005; 66(6): 830-834.

25. Imran M, Abida, Khan SA. Synthesis and Antimicrobial Activity of Some 2-Amino-4-(7-Substituted/Unsubstituted Coumarin-3-yl)-6-(Chlorosubstitutedphenyl) Pyrimidines. Trop J Pharm Res 2015; 14(7): 1265-1272.

26. Abida, Alaqel S, Imran M, El-Feky SA, Khan SA. Synthesis and antimicrobial activity of 3(substitutedphenyl)-1-(7-substitutedcoumarin-3-yl)prop2-ene-1-ones. J Chem Pharm Res 2013; 5(12): 10891093.

27. Barry AL. Procedures and theoretical considerations for testing antimicrobial agents in agar media. In: Lorian $\mathrm{V}$, Ed. Antibiotics in Laboratory Medicine, 3rd edn. Baltimore, Williams \& Wilkins; 1991; p 1-16.

28. Varma RS, Khan ZK, Singh AP. Eds. Antifungal agents: past, present, future prospects. Lucknow: National Academy of Chemistry and Biology, India, 1998; pp 55128. 